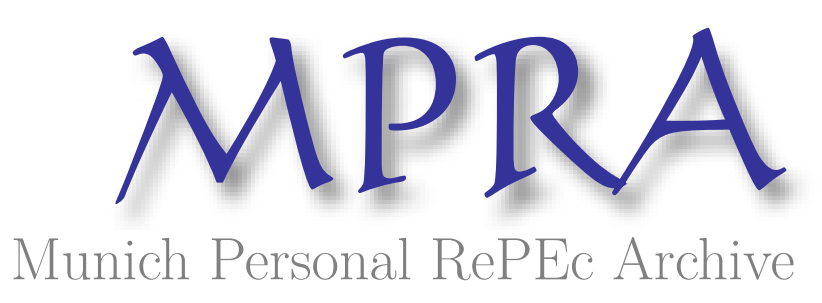

\title{
Securitization Structures and Security Design
}

Gauthier, Laurent

15 July 2019

Online at https://mpra.ub.uni-muenchen.de/95168/

MPRA Paper No. 95168, posted 18 Jul 2019 08:07 UTC 


\title{
Securitization Structures and Security Design*
}

\author{
Laurent Gauthier ${ }^{\dagger}$
}

First version: June 05, 2019, this version: July 15, 2019

\begin{abstract}
Securitization has been a subject of interest in the security design literature, and various models have been developed in order to explain why such transactions should produce senior securities and junior securities. However securitization structures are far more complex than a simple tranching by seniority. Using a model extending the existing literature, we derive new results by considering that interest and principal should be separately contractible, and that the senior bonds in a securitization should be par-priced, both realistic constraints. This allows us to derive optimal designs closely resembling actual securitization structures. Further, we show that the resecuritization of residuals, in the form of NIMs or reremics, is optimal through a pooling effect. We also analyze the interactions between collateral characteristics and pricing, reflecting securitization execution, and issuer structure choices. With a simple numerical application, we illustrate how important resecuritization is, and also how more attractive an excess-spread structure is relative to a more standard structure, as expected collateral losses increase. According to our analysis, the apparent complexity in excessspread structure and in resecuritizations can be explained by a valid optimal design argument.
\end{abstract}

Keywords: Securitization, security design, excess-spread, resecuritization

${ }^{*}$ This research was conducted while the author was a visiting professor at the University of Konstanz, Department of Economics, Institute of Finance.

${ }^{\dagger}$ Institute of Finance, University of Konstanz, PO Box 134, 78457 Konstanz, Germany, email: laurent.o. gauthier@gmail.com 


\section{Introduction}

Securitization structures, especially in the mortgage-backed securities market, are renowned to be particularly complex. This complexity has sometimes been credited as one of the contributing causes to the mortgage crisis of the late 2000s. Structuring, however, is presumably optimal, as a body of existing literature has shown.

Indeed, given that an issuer needs to securitize some assets, the question of the optimal structure in which this securitization should take place has been studied in the literature. It is directly related to the field of security design in corporate finance: the conception of an optimal financing security which will not be mispriced by investors. Debt, and on the other side leveraged equity, are specific types of securities or contracts; why do they exist in the form they have? Following Modigliani Miller, this issue has received extensive attention in economics and finance over the past 60 years. The optimality of particular securities is generally considered to be a consequence of information asymmetries. The models focused on security design tend to make specific assumptions about issuers and investors, so that the issuers have to issue securities and then one can ask what the optimal form for these securities should be. Several papers have focused on explaining why a simple debt/equity structure was optimal. For example, Boot and Thakor (1993), DeMarzo and Duffie (1999) and Dang, Gorton, and Holmström (2015), which use information asymmetry between issuers and investors or among investors, building upon the original work by Leland and Pyle (1977) and Myers and Majluf (1984). The right balance between debt and equity can be struck when there are information asymmetries. Empirical evidence on the design of structured securities as debt rather than equity is discussed in Begley and Purnanandam (2017), in Park (2013) for mortgages and in Franke, Herrmann, and Weber (2012) for CDOs.

Research has also shown there were various types of market imperfections that could justify more complex structuring, among them asymmetric information, market incompleteness, and transaction costs. Looking at the optimal design of debt contracts, Diamond (1993) showed that both maturity and seniority could be optimally engineered in order to alleviate the negative effects of information asymmetries, but in a context including refinancing and control takeover that is not applicable to securitized products. DeMarzo (2005) also considered information asymmetry and analyzed both pooling and tranching: through pooling, risk is diversified, and by tranching the bonds are made more informationally insensitive. Plantin (2003) focused only 
on tranching, but showed through an endogenous bidding mechanism that multiple seniority tranching is optimal when investors have diverse degrees of sophistication. In Gaur, Seshadri, and Subrahmanyam (2003) the authors considered market completeness and show that both pooling and tranching, by completing the market, accrue value to issuers and investors. Fender and Mitchell (2009) follow a different approach, and consider pooling and tranching from the perspective of the issuer holding a retained tranche, under information asymmetry. Using a project monitoring model similar to Gorton and Souleles (2007), Bougheas (2014) shows how a bank has an incentive to hold both an equity/junior exposure and part of a senior exposure, thus creating mezzanine debt that is sold to investors.

Actual securitization deals use a wide range of complex structural mechanisms, such as excessspread, over-collateralization, shifting-interest, interest or principal-only securities, triggers, resecuritizations in the form of net interest margin structures or reremics, and many other features. These features have rarely been specifically studied, and in particular the optimality of their design has not been looked into. One exception is Hein (2009) who carried out a numerical analysis showing how a reserve mechanism can greatly improve the efficiency of senior/subordination. In this paper we show that many seemingly complex features actually do serve a purpose, and are optimal within the framework of existing security design models.

The paper is organized as follows: the next section gives an overview of the salient features in securitization structures, neglecting certain aspects of dynamic cash flow allocation and focusing on the main characteristics observed in these transactions.

The third section introduces a model, based on DeMarzo and Duffie (1999) and on DeMarzo (2005), in which the optimal security design applicable to securitizations can be analyzed. New results are derived, showing the optimality of a senior/subordination structure paying a pro-rated coupon. The fourth section looks into the reasons for more complex structures, namely the use of excess-spread and resecuritization. Under the constraint of par-priced senior bonds, using excess-spread is shown to be optimal over a pure senior/subordination structure. In addition, resecuritization is also shown to be optimal at the limit, offering a justification for structures such as NIMs and reremics.

In the fifth section, the impact of a securitization exit on loan pricing is considered and a numerical analysis shows how excess-spread structures are preferred on low quality collateral, over more standard senior/subordination structures. 
The sixth section concludes, and is followed by the Appendix in the seventh section, and references in the eighth.

\section{Securitization Structures}

There are in fact no general concepts in the structuring of securitizations, as the design of every deal will potentially be tailored to the very specific requests from investors or rating agencies. The broad notions we discuss here remain approximations, but they capture many of the salient aspects of these bonds, and enough of their complexity.

In the finance and economics academic literature detailed overviews of securitization structures are rare. One, specifically focused on credit enhancement structures in the non-agency market covering both standard structures (so-called "six-packs") and excess-spread/over-collateralization structures can be found in Gorton (2008). Practitioners manuals such as Fabozzi (2016), or investment bank reports such as Crawford (2007), Hayre and Young (2004), Gauthier (2004), Gauthier and Zimmerman (2002b), Gauthier and Zimmerman (2002a) and Gauthier (2002) provide more detailed descriptions and analyzes. See McConnell and Buser (2011) for a history of the MBS market across the spectrum from prime to subprime.

We begin by discussing the allocation of interest in securitization structures. In the following subsection we explain the inner workings of two very common structures in the private-label mortgage securitization market: six-packs or shifting-interest/senior-subordination structures (SI/SS), and excess-spread/over-collateralization structures (XS/OC). Then, we look into the resecuritization of the residuals from these SI/SS or XS/OC structures into reremics and net-interest margin securitizations, or NIMs.

\subsection{Interest Allocation and Par-Pricing}

One particular aspect of securitization structures is that one of the main drivers of structuring logic is the need for par-pricing on senior securities. The coupon on the bonds is normally determined so that the senior bonds are priced at par at issuance (the coupon on them is equal to the required net yield).

In the case of corporate bond or loan issuance, this may seem as a trivial consideration because setting the coupon to a particular level that makes the bond par priced is indeed only a question 
of "writing it down" in the debt contract. In the case of securitization structures the matter is different because the underlying assets have their own set characteristics, and one cannot simply set the coupon on senior bonds to an arbitrary amount. Hence, there is a significant effort in structure design specifically aimed at manufacturing par-priced bonds. This implies allocating interest in relation to principal and expected losses, so that the bonds' coupon exactly matches their required net yield.

Excess-spread (typically accompanied with over-collateralization, as we will see further below) as well as interest stripping (making interest- or principal-only bonds) are the usual structural methods of choice to build par-priced bonds. Looking at the range of securities typically issued in a securitization deal, there is as much complexity allocated to the manufacturing of par-priced bonds as there is to creating senior and subordinated bonds.

\section{$2.2 \quad$ Six-Packs and Excess-Spread}

In securitizations, the senior securities issued are always in the form of bonds, and hence have a face value and pay a coupon. This is in fact true even if the underlying assets are not loans and do not have a principal attached to them, such as in the case of the securitization of royalties or franchise payments, for example. The coupon is a given proportion of the outstanding balance, typically paid monthly.

The simplest structures need to be based on collateral that separately pay principal and interest, as is the case with a portfolio of mortgage loans. These structures treat the allocation of interest and principal separately: interest collected from the collateral is used to pay interest on the bonds, and principal collected from the collateral is used to pay principal on the bonds.

The credit protection that makes the senior bond more resilient to collateral losses than the other securities in the deal comes from the subordinated bonds which are allocated losses in priority. There are typically six of them, with ratings ranging from AA to a first-loss non-rated tranche, hence the "six-pack" nickname. The subordinated bonds are all subordinated to each other in order of seniority.

In addition to specifying this particular subordination ordering, the structure addresses the timing of principal payments. Indeed, without further specification, prepayments would be distributed on a pro-rata basis among all the outstanding bonds, and this would partially retire the subordinated bonds potentially before they are needed. Hence, the principal payments to the 
subordinated bonds should also be structured so that they are delayed until the senior bonds are fully repaid. This would ensure that the entirety of each bond in the six-pack would be available to provide protection to the senior bonds. However, relative to this extreme solution, structures in the Jumbo and Alt-A markets have for decades contained a special feature, shifting-interest principal allocation, which effectively releases some principal to the subordinated stack before the senior bonds are repaid.

The simplest structure observed in the non-agency market is hence shifting-interest/seniorsubordination with an interest-only tranche collecting extra interest with which the senior bond would otherwise be priced above par. This is represented in Figure 1.

[Figure 1 about here.]

In cases where the collateral pays a coupon that is much higher than the yield required by the market on a typical AAA-rated senior bond, a different kind of structure has been used since the mid-1990s: excess-spread ("XS"). The higher expected losses on subprime or the low-end of the Alt-A market lead to these loans paying a high coupon. This additional interest cash flow, relative to prime collateral, can be used to further improve credit enhancement, through the excess-spread structure: some amount of interest is used in order to repay principal on the bonds faster. By doing so, the structure can withstand higher collateral losses: while the collateral balance is reduced by principal losses, it does not have to translate into a writedown for the senior bonds as far as the interest cash flow was used to reduce the bond balance by the same amount. Excess-spread designates the use of the interest cash flow that is not necessary to pay interest on the bonds, which would otherwise have gone to an interest-only tranche in the standard structure discussed earlier.

Without further specification, excess-spread would be paid out to some tranche holder at times when it is not put to use to absorb losses. This raises a problem: excess-spread is likely to be maximal early in the life of a deal, when the collateral balance is highest and losses have not yet ramped up, but would probably be needed later when loans are most likely to default. In order to capture excess-spread and put it aside, an additional feature called over-collateralization ("OC") is used: a large share of excess-spread is used to pay down principal on the senior bonds, even though there may not be any losses. By paying down the balance of the bonds faster than it would normally be paid down with collateral principal cash flows, a imbalance is created: there is less principal outstanding on the bonds than there is on the collateral. The difference is, literally, 
over-collateralization. This over-collateralization then effectively behaves like a subordinated tranche.

In a large majority of MBS transactions making use of that technique, over-collateralization is not entirely built dynamically by collecting available excess-spread, but is set to a certain amount at issuance. This amount is called the upfront OC, and typically varies with the collateral's credit risk. This upfront OC is just the difference between the collateral principal balance and the bonds principal balance at the deal's issuance, and is akin to a first-loss, deeply subordinated bond (although it is not debt, since it does not have a stated principal balance).

As in the case of the subordinated bonds in a shifting-interest structure, which receive some principal payments earlier than before the senior bonds are fully paid off, the over-collateralization tranche typically receives some cash flows before the end of the deal. This earlier payment is called an OC release.

Figure 1 also shows a simplified representation of an XS/OC structure. One can see how the extra interest is in fact used to build OC, as opposed to being diverted to an IO tranche holder. The figure also shows that the XS/OC is not the only credit-enhancement structural mechanism in a typical deal structure, as the bonds block also benefits from some subordination. In effect, the senior bonds are protected by a combination of excess-spread, over-collateralization, and subordinated bonds.

\subsection{Reremics and NIMs}

Across the spectrum of non-agency mortgage-backed securities, from prime to subprime, and irrespective of the initial structural design choices, the junior-most tranches have been resecuritized. This pattern has been most pronounced and most visible in the subprime market, for the simple reason that the junior securities were much larger. In the jumbo prime market, the size of the six-pack is small, often less than $2 \%$ of the entire deal balance, and the non-investment grade part (BB-rated and below) less than $0.5 \%$. In subprime, the non-rated excess-spread and over-collateralization residual and junior-most non-investment grade bonds amount to $6-8 \%$ of the initial deal balance.

The junior-most securities in a structure, such as the bottom of the six-pack or the XS/OC tranches, have typically been retained by deal issuers, but only for a limited amount of time in many instances. Indeed, once enough of these residuals could be accumulated, they have been 
resecuritized in simple front cash flow structures.

In the prime and clean Alt-A markets, this resecuritization has taken the form of a "reremic"1: very junior tranches grouped as a portfolio, off of which a senior security is issued, receiving all cash flows in priority. This structure itself effectively makes use of excess-spread: any cash flow collected from the portfolio of junior securities, whether it be principal or interest, is used to repay interest and principal on the senior bond as needed. Figure 2, taken from an investment bank presentation, shows a graphical depiction of a reremic.

[Figure 2 about here.]

In the subprime market and lower end of the Alt-A market where XS/OC structures were the norm, the resecuritization of residuals took the form of a net-interest margin securitization ("NIM"). The name comes from the fact that a large part of the cash flows comes from the difference between two interest streams: that collected on the collateral and that due to the bonds. Figure 3 shows a very simple depiction of a NIM.

[Figure 3 about here.]

The existing literature on security design has explained why there may be senior and junior bonds, and why there could be multiple layers of such securities, but the specificity and complexity of the actual securitization designs discussed above do not seem to match the highly stylized optimal structures from existing models.

We will strive to explain the following features in securitization transactions, which so far have not been explained by the security design literature:

- Interest and principal are differently allocated and not commingled in some cases,

- The issuers who retain residuals actually obtain some senior cash flows in the form of OC releases or earlier principal payments to deeply subordinate bonds

- In some cases (in particular low credit quality collateral), interest and principal are commingled, and a structure design choice of XS/OC versus SI/SS takes place,

- The senior bonds need to be par-priced, and the structure needs to be designed accordingly.

\footnotetext{
${ }^{1}$ Literally, the re-application of a Real Estate Mortgage Investment Conduit legal structure
} 


\section{Optimal Debt Structure for Securitization}

Maybe the most fundamental discrepancy between the security design models we briefly discussed earlier and the structuring logic summarized in Section 2 is the fact that interest and principal are very systematically segregated in many structures. The model by DeMarzo and Duffie, which has a very complete representation of the underlying collateral noise and information structure, considers cash flows as an aggregate. In consequence, the structuring is applied to the total cash flow, and the typical optimal debt-like contract would commingle all interest payments with the principal. Since this is quite different from standard structuring logic, it makes sense to look closer into the application of security design models to cash flows more closely representative of a loan portfolio.

In order to capture differential behaviors between interest and principal, we choose to model a random coupon, and collateral losses separately. The coupon's randomness may come from the fact it is indexed on an external reference, such as 3-month Libor. We assume that the coupon as a random variable is independent from the credit performance of the loans. This is a reasonable assumption because the dynamics of coupon indices are typically not strongly related to the credit performance of a particular pool of loans. In addition, the coupon has a multiplicative effect on the principal returned: indeed, the loans that default do not pay their interest, so credit losses effectively lower the total amount of interest recovered.

\subsection{Framework and Assumptions}

The collateral balance is set to 1 for simplicity, and the portfolio maturity is fixed at time 1 . The underlying losses $L$ are assumed to follow a distribution with a compact support included in $[0,1]$. The coupon $c$ is a positive random variable. The interest payment comes from the coupon rate $c$ and is made at maturity. Since there may be credit losses and the loans that default do not pay a coupon, the total cash flow from the collateral is: $Y=(1-L)(1+c)$.

We consider we are in the framework of the security design model from DeMarzo and Duffie (1999): an issuer with a higher retention cost than investors and with information on the assets' credit risk is looking to sell optimal securities. We assume that the issuer observes information that conditions $L$ privately, as it can access information relevant to the quality of the loans, but does not know $c$ in advance. Although the issuer of loans may be aware of or control the some of the drivers of the quality of the loans, it cannot control or know in advance the value of interest 
rate indices. While the model from DeMarzo and Duffie can be extended to account for a non zero discount rate for investors, it makes calculations more extensive and does not add to our analysis. Hence, as in their original model, we assume that investors do not discount cash flows, and the issuer discounts them at a rate $y$. To investors, without discounting, the value of an asset paying principal $1-L$ and coupon $c$ is $\mathbb{E}[(1+c)(1-L)]$.

In the model by DeMarzo and Duffie, the entire cash flow $Y$ is the contractible variable, and it is only assumed that $Y$ is not independent from the privately observed $Z$. In order to account for the distinction between interest and principal payments, we will write $Y=X V$ where $X=(1+c)$ is the interest multiple, $X$ is independent from $V$ and $X \geq 1^{2}$. The variable $V=1-L$, representing the principal recovered, is assumed to possess a conditional distribution $\mu_{V}(v, z)$ relative to $Z$, that is continuous as a function of $z$. Also, we assume that $V \in[0,1]$, as one cannot recover more than the initial balance.

The extension of the model from DeMarzo and Duffie to an investor specific discount rate, along with a random payment time $T$ independent from $Z$ but not independent from $Y$ is difficult, because then the cash flows evaluated by the issuer would have the form $g(Y)(1+y)^{-T}$. This makes the extension of the model much more complex and potentially intractable. However, the simple random coupon approach we follow can represent the maturity randomness if the investors' discount rate is null: we can simply consider that the cash flows have the form $V(1+c T)$ where $c$ may be a random coupon, and $T$ is a positive continuous variable representing the random maturity. The issuer's discounting of the future cash flows is assumed to be independent from the time over which the cash flows take place, for example if all interest payments to the issuer are kept in escrow until a legal maturity equal to the maximum possible $T$. Also note that the time $T$ may represent the average life of cash flows taking place over time, rather than a maturity at which a full bullet payment takes place. The fact that the issuer does not have any privileged information on timing, but only on credit, is consistent with the notion that prepayments may be driven by factors independent from the issuer's control, unlike loan origination.

One important technical aspect is that we assume that $V$ has a uniform worst-case $z_{L}$ : there exists a value $z_{L}$ and an increasing function $\nu_{z}(v)$ such that for all measurable $g$,

$$
\int g(v) \mu_{V}(v, z) d v=\int g(v) \nu_{z}(v) \mu_{V}\left(v, z_{L}\right) d v .
$$

\footnotetext{
${ }^{2}$ we are assuming investors do not discount cash flows, so implicitly we are requiring that the collateral coupon is higher than the yield required by the market.
} 
Because of this, for any increasing function $g, z_{L}$ is the minimum argument of $\inf _{z} \mathbb{E}[g(Y) \mid Z=z]$. We also assume that $Y=X V$ has the same uniform worst-case $z_{L}$.

A most important extension of the original model is to consider that interest is contractible separately from principal. In other words, a security design can be expressed as $g(X, V)$ rather than $g(X V)$. We will assume that all security designs we consider are continuous and increasing (in $y$ or in both $x$ and $v$ ), and in all cases $g(Y) \leq Y$.

As in the model by DeMarzo and Duffie, the issuer decides on an optimal design before being able to observe $Z$ or the other variables, although the distributions of all the random variables are known in advance.

We note $V(g(Y))$ for the value to the issuer of a particular security design, following DeMarzo and Duffie, with

$$
V(g(Y))=\frac{y}{1+y} \mathbb{E}\left[g(Y) \mid Z=z_{L}\right] \mathbb{E}\left[\left(\frac{\mathbb{E}\left[g(Y) \mid Z=z_{L}\right]}{\mathbb{E}[g(Y) \mid Z]}\right)^{\frac{1}{y}}\right]
$$

where we use the fact that $\mathbb{E}\left[g(Y) \mid Z=z_{L}\right]=\min _{z} \mathbb{E}[g(Y) \mid Z=z]$. Note that $V$ is meant as a functional of $g(Y)$, not as a function of a particular $g(Y)$-measurable outcome.

\subsection{Dynamic Rather than Standard Debt}

Standard debt can be defined as a particular security design of the form $g(y)=y \wedge D$, for a face value $D$. It has been shown to be an optimal security design under some particular conditions. We define dynamic debt with face value $D$ as a design of the form $g(x, v)=f(x)(v \wedge D)$; the term dynamic meaning that the actual total cash flow that is paid is random even if the full face value is repaid.

Standard debt of the form $g(X V)=X V \wedge D$ goes against the grain of practice in securitization, because it commingles interest and principal cash flows, in such a way that interest may effectively compensate for a shortfall in principal, and principal may compensate for a shortfall in interest. This is very different from the typical designs in securitizations, where in most cases interest is paid in a prorated fashion relative to the expected principal payments. In effect, designs in the form of dynamic debt are much more common in securitization than standard debt, as defined in DeMarzo and Duffie (1999) or DeMarzo (2005). 
We will see, under our assumptions, that if interest is contractible then a standard debt contract is not optimal, but rather a dynamic debt contract allowing for a pro-rated coupon payment of the form $g(x v)=x(v \wedge D)$ for a face value $D$.

We have the following proposition:

Proposition 3.1 (Optimality of Dynamic Debt). Among all contracts of the form $g(x v)$, standard debt $g(x v)=x v \wedge D$ is optimal for some $D$. Among all contracts of the form $g(x, v)$, which include those of the form $g(x v)$, dynamic debt $g(x, v)=x(v \wedge D)$ is optimal for some $D$, and therefore dominates standard debt.

The proof is in the Appendix.

Allowing for the specific contracting of interest cash flows, we therefore find that the optimal security design is consistent with the structures observed in securitization. Prorated interest is paid to the senior bonds.

We note:

$$
m(D)=\mathbb{E}\left[V \wedge D \mid Z=z_{L}\right] \mathbb{E}\left[\left(\frac{\mathbb{E}\left[V \wedge D \mid Z=z_{L}\right]}{\mathbb{E}[V \wedge D \mid Z]}\right)^{\frac{1}{y}}\right] .
$$

We will then note $D^{*}$ the optimal face value that maximizes $m$ :

$$
D^{*}=\arg \max _{D} m(D) .
$$

This optimal $D^{*}$ is a function of $y$ and of the distribution of $V$ and $Z$, and is the optimal face value that maximizes the value of the dynamic debt contract $x(v \wedge D)$ for the issuer. We will write the optimal dynamic debt contract $g_{P R}(x, v)=x\left(v \wedge D^{*}\right)$. Also for simplicity we write $m=m\left(D^{*}\right)$ since $D^{*}$ depends on the same inputs as $m$.

Note that the senior tranche is not entirely sold to investors, and the issuer retains part of it. The quantity sold, according to Proposition 2 in DeMarzo and Duffie (1999), is expressed as

$$
Q^{*}\left(\mathbb{E}\left[g_{P R}(X, V) \mid Z\right]\right)=\left(\frac{\mathbb{E}\left[V \wedge D^{*} \mid Z=z_{L}\right]}{\mathbb{E}\left[V \wedge D^{*} \mid Z\right]}\right)^{\frac{1+y}{y}}<1 .
$$


For convenience, we will write the expected quantity as:

$$
\mathbb{E}\left[Q^{*}\left(\mathbb{E}\left[g_{P R}(X, V) \mid Z\right]\right)\right]=q,
$$

since $D^{*}$ depends on the same inputs as well. The expected price corresponding to this quantity is

$$
\mathbb{E}\left[P^{*}\left(Q^{*}\left(\mathbb{E}\left[g_{P R}(X, V) \mid Z\right]\right)\right)\right]=\mathbb{E}\left[\mathbb{E}\left[g_{P R}(X, V) \mid Z\right]\right]=\mathbb{E}\left[g_{P R}(X, V)\right]=p \mathbb{E}[X],
$$

where $p=\mathbb{E}\left[V \wedge D^{*}\right]$. Both $q$ and $p$ as expectations represent either the investors' estimates or the issuer's own estimates before knowing the private information $Z$.

We can make some further assumptions to potentially simplify the expression of the optimal securities, but they are not essential to obtaining our results. In particular, it is reasonable to assume that the the principal amount $V$ is the product of two independent variables: $V=W Z$, where $Z$ is the issuer's private information, and $W$ represents the common information on credit risk. We assume that $W \in\left[w_{L}, 1\right]$ and $Z \in\left[z_{L}, 1\right]$. Using a multiplicative relationship to represent private information is consistent with the issuer's knowledge of credit risk being expressed in odds ratios: the characteristics of a particular portfolio of loans only known to the issuer may make it worse or better than the typical portfolio.

We can characterize how to satisfy the uniform worst-case condition when the cash flows behave multiplicatively relative to the issuer's information, as we have assumed above.

Proposition 3.2 (Conditions for Uniform Worst Case). If the distribution $\mu_{A}$ of a random variable $A$ is log-concave and such that $\frac{\mu_{A}^{\prime}(a)}{\mu_{A}(a)}$ vanishes at zero, or if $\frac{\mu_{A}^{\prime}(a)}{\mu_{A}(a)}$ goes to infinity and $\frac{\mu_{A}^{\prime}(a)}{\mu_{A}(a)} \geq \alpha \frac{\mu_{A}^{\prime}(\alpha a)}{\mu_{A}(\alpha a)}$ for $\alpha \geq 1$, then the uniform worst case of $A Z$ is $z_{L}$.

The proof can be found in the Appendix.

We can apply this proposition to $A=W$ and $A=W X$, in which case $V$ and $Y$ admit a uniform worst-case $z_{L}$, if $W$ and $W X$ verify one of the the conditions of the proposition.

For example, the distribution of density $\mu_{A}(a)=(\beta+1) a^{\beta}$ on $[0,1]$ satisfies the second condition. A uniform distribution satisfies the first condition. 


\section{Reasons for More Complex Structures}

\subsection{Targeted Coupon, Strip Down and Excess-Spread}

We can now see to what extent more complex structures such as stripping or excess-spread may be optimally used. In the optimal designs we have discussed so far, we have not addressed an important requirement of structured securities: they need to have a specific coupon. In particular, structured securities are typically issued at par, so that their initial valuation equals their face value. In other words, they are designed so that the expected coupon they pay exactly compensates for the discount rate demanded by investors. In our simple construct we are assuming that investors do not have a discount rate, so we are setting the structural requirement in terms of a coupon target: an interest rate such that the expected value of the cash flows capitalize at that rate.

In order to properly define the notion of coupon on a structured security, we need to also define its principal. Given any design $g$ such that $g(x, v)=f(x, v) k(x, v)$ and $f(x, v) \geq 1$, we call $f(x, v)$ the coupon and $k(x, v)$ the principal.

The requirement for a specific expected coupon $s$ implies that a security design $g=f k$ must verify

$$
\mathbb{E}[f(X, V) k(X, V)]=(1+s) \mathbb{E}[k(X, V)] .
$$

We are interested in the most common case in securitization, where the coupon collected on collateral is greater than the coupon required by investors, so we assume that $1+s<\mathbb{E}[X]$.

We will also need to define the notion of excess-spread more formally. The standard structuring method, without the use of excess-spread, allocates principal and interest separately: the interest paid on the structured bond comes from the collateral's interest payment, and the principal paid on the structured bond comes from the collateral's principal payment.

A standard structure can therefore be defined as a security design $g$ such that $g(x, v)=f(x) k(v)$ with $f(x) \leq x$ and $k(v) \leq v$. In other words one cannot pay more interest or principal than the interest or principal, respectively, that is collected.

In contrast, an excess-spread structure allows the use of interest to repay principal, and reciprocally, and therefore does not have the particular restriction above: it can be defined as a security design $g$ such that $g(x, v)=f(x, v) k(x, v)$ with the only constraint that coupon is positive, so 
$f(x, v) \geq 1$.

Based on these definitions, we have the following proposition:

Proposition 4.1 (Optimal Standard Structure). With a target coupon of $s$, the standard structure $g_{S S(s)}$ paying principal of $V \wedge D^{*}$ and a coupon of $\frac{X(1+s)}{\mathbb{E}[X]}$ is optimal.

Proof. First, we can verify that a standard structure with a coupon as defined satisfies the condition for the target coupon. The design stated in the proposition is a standard structure of the form $g(x, v)=\frac{x(1+s)}{\mathbb{E}[X]} k(v)$. We have

$$
\mathbb{E}[g(X, V)]=(1+s) \mathbb{E}[k(V)]
$$

so this security effectively pays the target coupon.

Once the coupon condition is verified, the value of this design to the issuer only depends on $k$. Finding the optimal $k$ is equivalent to finding an optimal design structured off of a cash flow $V$. Since we have assumed that $V$ had a uniform worst-case $z_{L}$ (the same as $V X$ ), then we know thanks to Proposition 10 in DeMarzo and Duffie (1999) that the optimal design $k$ is of the form $k(v)=v \wedge D$ for some $D$ maximizing

$$
V(V \wedge D)=\frac{y \mathbb{E}\left[V \wedge D \mid Z=z_{L}\right]}{1+y} \mathbb{E}\left[\left(\frac{\mathbb{E}\left[V \wedge D \mid Z=z_{L}\right]}{\mathbb{E}[V \wedge D \mid Z]}\right)^{\frac{1}{y}}\right]
$$

We know that this expression is maximized by $D^{*}$, and the proposition is proved.

Under the constraint that a target coupon should be reached, and if one can only create standard structures, the common technique of stripping down the coupon is an optimal design.

However, this structure is not absolutely optimal. We can easily see that

$$
V\left(g_{P R}(X, V)\right)-V\left(g_{S S(s)}(X, V)\right)=\frac{y m}{1+y}(\mathbb{E}[X]-(1+s)),
$$

which is positive since $1+s<\mathbb{E}[X]$.

Using excess-spread will allow us to circumvent this issue of suboptimality, as the following proposition illustrates. 
Proposition 4.2 (Optimal Excess-Spread Structure). With a target coupon of $s$, the excessspread structure $g_{X S(s)}$ paying principal of $\frac{X}{1+s}\left(V \wedge D^{*}\right)$ and a coupon of $(1+s)$ is optimal. In addition,

$$
V\left(g_{X S(s)}(X, V)\right)=V\left(g_{P R}(X, V)\right)
$$

Proof. We can verify that the design respects the target coupon constraint: with the principal set to $k(x, v)=\frac{x}{1+s}\left(v \wedge D^{*}\right)$, we have

$$
\mathbb{E}\left[g_{X S(s)}(X, V)\right]=(1+s) \mathbb{E}[k(X, V)]
$$

In addition, $g_{X S(s)}(x, v)=x\left(v \wedge D^{*}\right)$ and hence $g_{X S(s)}=g_{P R}$, so we know that $g_{X S(s)}$ is optimal.

Whether the issuer creates a standard structure or uses excess-spread, the entire tranche is not sold. In both cases, the quantity sold is the same since it only depends on the distribution of $V$ and of $D^{*}$. We have

$$
Q^{*}\left(\mathbb{E}\left[g_{S S(s)}(X, V) \mid Z\right]\right)=Q^{*}\left(\mathbb{E}\left[g_{X S(s)}(X, V) \mid Z\right]\right)=Q^{*}\left(\mathbb{E}\left[g_{P R}(X, V) \mid Z\right]\right) .
$$

In expectation, all these quantities are equal to $q$.

The total cash flow retained by the issuer can be seen equivalently as collateral minus $q$ shares of senior bonds, or 1 unit of subordinated cash flow plus $1-q$ units of senior bond. The share of senior cash flows that is retained by the issuer can be paralleled with the typical principal return mechanisms that exist for subordinated tranches in securitization structures, which we presented in Section 2:

- In the case of standard structures, subordinated bonds are normally not pure last cash flow sequentials, and receive some early principal payments. The subordinated bonds are said to deleverage in this case. Such features may be called two-times tests, or turbo payments.

- In the case of excess-spread structures, the excess-spread residual retained by the issuer is typically paired with an over-collateralization tranche acting as a kind of subordinated tranche, which can also receive an over-collateralization release: an early principal payment. 
The share of senior cash flow retained by the issuer effectively works as such a release of safe principal cash flow.

Let us now see how both standard and excess-spread structures may coexist in the same market. The targeted coupon can be set so that the securities are par-priced, which with our assumption regarding the investors' discount rate translates into $s=0$. Also, in order to capture the more common reasons for randomness in total coupon cash flow, we consider that the coupon rate $c$ is fixed, but the timing is random, so that $X=(1+c T)$ for some positive variable $T$ (such that the assumptions on $X$ are still valid).

In this case, the difference between the issuer's gain using an excess-spread structure and a standard structure, if securities have to be par priced, is:

$$
V\left(g_{X S(0)}(X, V)\right)-V\left(g_{S S(0)}(X, V)\right)=\frac{c y \mathbb{E}[T] m}{1+y} .
$$

Since an excess-spread structure uses interest to pay principal, obtaining a rating would be expected to require more work, as the analysis of interest payments needs to be factored in, although in the case of a standard structure it is not necessary. As a result it is reasonable to assume there is a cost $k$ in executing such a structure. We consider this cost, paid by the issuer, to be proportional to the entire collateral balance (set to 1).

As a consequence, the issuer will choose to execute an excess-spread structure if

$$
V\left(g_{X S(0)}(X, V)\right)-V\left(g_{S S(0)}(X, V)\right)>k .
$$

This condition simplifies into:

$$
c>\frac{k(1+y)}{y \mathbb{E}[T] m} .
$$

Hence, for low collateral coupons, we will expect to see standard structures, while high collateral coupons will lead to excess-spread structures. This is exactly consistent with the patterns that have been observed in securitization structures. In the prime market, securitizations are mostly based on standard structures, in the subprime market mostly on excess-spread structures, and in the Alt-A market both have been observed. 


\subsection{Reremics and NIM Structures}

Net-interest margin structures have been quite common in the subprime market, and to a lesser extent in the Alt-A market. They are applied to the residual of an excess-spread securitization, which contains a stream of interest commingled with some principal. More standard resecuritizations, reremics, in the Alt-A and prime market have taken the form of a securitization of a basket of deeply subordinated bonds.

Since a reremic or a NIM is a further securitization of the cash flows normally kept by the issuer in the first place, we should be able to explain how a presumably optimal contract, the original structure, could be further improved. We know that NIM securitizations have for the most part been backed by several underlying residuals. Typically, NIMs combined 2 or 3 separate residuals, sometimes more, from similar deals, and not always the entirety of the residual cash flows. In the case of the resecuritization of subordinated bonds, the portfolios usually contained tens of securities.

We considered that the issuer designed the initial optimal excess-spread structure $g_{X S}(X, V)=$ $X\left(V \wedge D^{*}\right)$ ex-ante, before gaining access to the private information $Z$. At the time the structure is sold, the investors will be able to observe the amount issued $Q^{*}(Z)$, which is a function of the private information. In particular, if $\mathbb{E}\left[Y \wedge D^{*} \mid Z=z\right]$ is increasing as a function of $z$, investors will recoup the private information $Z$ from the observation of the quantity of bonds for sale.

In order to capture the logic in NIMs or reremics, we will assume there are $n$ deals being structured, all in the same sector with the same distributional characteristics $Y_{i}=X_{i} W_{i} Z_{i}$ with $0<i \leq n$, where there is the same uniform worst case $z_{L}$ for all $Y_{i}$. The $X_{i}, W_{i}$ and $Z_{i}$ are all independent with the same distributions as $X, W$ and $Z$. We have assumed that $X$ and $W X$ verified the conditions of Proposition 3.2, but we will further assume that $X W Z$ is log-concave, so that the uniform worst-case property is stable by summing variables of the form $X_{i} W_{i} Z_{i}{ }^{3}$. We will also consider that we can focus on NIMs only, since in the context of our model the only difference with a reremic would be by a scalar (the residual being larger in the case of a standard structure).

The issuer observes all the $Z_{i}$ and the deals' optimal structures are the same $D^{*}$. The tranches have not yet been sold, so that investors are not yet aware of the optimal quantities selected by

\footnotetext{
${ }^{3}$ See DeMarzo (2005)
} 
the issuer for each deal $Q^{*}\left(Z_{i}\right)$. For each deal, the issuer retains the following cash flow, which is a combination of a slice of collateral and excess-spread structure residual:

$$
C_{i}=\left(1-Q^{*}\left(Z_{i}\right)\right) X_{i}\left(W_{i} Z_{i} \wedge D^{*}\right)+X_{i}\left(W_{i} Z_{i}-D^{*}\right) \mathbb{I}_{W_{i} X_{i}-D^{*} \geq 0}
$$

The first term in this expression is the retained senior exposure, and the second term is the residual subordinated exposure. Since the retained senior exposure is not known at the time when the structure is created (before observing private information), it cannot be used as part of the NIM structure, and only the residual $R_{i}=X_{i}\left(W_{i} Z_{i}-D^{*}\right) \mathbb{I}_{W_{i} Z_{i}-D^{*} \geq 0}$ is used to create a NIM.

Our approach is similar in philosophy to that of DeMarzo (2005), with two fundamental differences:

- Our private information $Z$ has a multiplicative effect on the underlying risk $W$ while in DeMarzo's paper it is additive, and the additivity simplifies some aspects of the limiting behavior

- We are considering the securitization residual, not the assets themselves, which also makes the analysis more complicated.

Creating a NIM off of each deal separately cannot be optimal. Indeed, this would simply be equivalent to altering the initial structure, which we have shown to be optimally designed as $g_{X S}(x, v)=x\left(v \wedge D^{*}\right)$. As a result a single NIM defined as $g_{N I M}(x, v)=f\left(x\left(v-D^{*}\right) \mathbb{I}_{v-D^{*} \geq 0}\right)$ for some design $f$ would only be optimal for $f=0$. We will show that by pooling different residuals together, the NIM resecuritization further improves the issuer's value.

Since a NIM is based off of an excess-spread structure, we can assume that it is possible to commingle interest and principal as needed in order to create par-valued securities, just as we did in the case of senior bonds relying on excess-spread.

Proposition 4.3 (Optimality of NIM Resecuritization). Creating a NIM structure off of a series of excess-spread residuals further improves the issuer's value. At the limit the optimal structure is

$$
g_{N I M}\left(\frac{1}{n} \sum_{i=1}^{n} R_{i}\right)=\left(\frac{1}{n} \sum_{i=1}^{n} R_{i}\right) \wedge \mathbb{E}\left[\left(z_{L} X W-D^{*}\right) \mathbb{I}_{z_{L}} X W-D^{*} \geq 0\right],
$$


and the value to the issuer verifies

$$
\lim _{n \rightarrow \infty} V\left(g_{N I M}\left(\frac{1}{n} \sum_{i=1}^{n} R_{i}\right)\right)=\frac{y}{1+y} \mathbb{E}\left[\left(z_{L} X W-D^{*}\right) \mathbb{I}_{z_{L} X W-D^{*} \geq 0}\right]
$$

The proof is shown in the Appendix.

Resecuritization appears as an efficient way of exploiting diversification to further increase the issuer's value.

\section{Impact of Securitization on Collateral Valuation}

\subsection{Formal Analysis}

Without a NIM securitization, the issuer creates senior bonds, necessarily priced at par, and retains the junior securities and some of the senior bonds. This issuer may originate the loans itself or buy them, but in any case we will assume that there are many such issuers in competition and therefore, at a marginal profit of 0 , the valuation of the loans is driven by the valuation of the structured securities that are issued.

We consider the valuation before the issuer knows the private information $Z$, since potential choices between different types of collateral would have to be made before the specifics are known. For example, an issuer may choose to concentrate on prime borrowers rather than subprime borrowers before the details of a particular prime loan portfolio that the issuer originates are known. Also note that we implicitly assume that the investors do not have access to this primary market, but must go through the issuer.

The valuation of the collateral based on a securitization execution can be written as follows, in the two cases depending on the type of structure satisfying the par valuation requirement:

$$
P_{X S}=\left(q+\frac{1-q}{1+y}\right) p \mathbb{E}[X]+\frac{\mathbb{E}[V]-p}{1+y} \mathbb{E}[X],
$$

and

$$
P_{S S}=\left(q+\frac{1-q}{1+y}\right) p+\frac{\mathbb{E}[V] \mathbb{E}[X]-p}{1+y}
$$


The difference can be written

$$
P_{X S}-P_{S S}=(\mathbb{E}[X]-1) \frac{1+q y-p}{1+y},
$$

which is positive since $\mathbb{E}[X]>1$ and $p \leq 1$.

The collateral we consider is a portfolio of loans, and when these loans are made they typically are valued at par. Also recall that we can write $X=1+c T$. Therefore, there are coupons $c_{S S}$ and $c_{X S}$ such that $P_{S S}=1$ and $P_{X S}=1$. Solving for these coupons and after some simplifications we find:

$$
c_{S S}=\frac{1+y-p q y-\mathbb{E}[V]}{\mathbb{E}[V] \mathbb{E}[T]}
$$

and

$$
c_{X S}=\frac{1+y-p q y-\mathbb{E}[V]}{(p q y+\mathbb{E}[V]) \mathbb{E}[T]} .
$$

All else being equal, we can see that the collateral coupon if an excess-spread structure is used is lower than if a standard structure is used. In both cases, a longer expected time to repayment leads to a lower coupon.

Both par coupons could be compared to the par-coupon obtained if the collateral was valued by the issuer under the assumption it did not result in a securitization. In this case, the collateral would be worth

$$
P_{I}=\frac{\mathbb{E}[X] \mathbb{E}[V]}{1+y},
$$

and solving for the par coupon gets us

$$
c_{I}=\frac{1+y-\mathbb{E}[V]}{\mathbb{E}[V] \mathbb{E}[T]} .
$$

As could be expected, the par coupon is higher in the case when there is no securitization execution: $c_{I} \geq c_{X S} \geq c_{S S}$.

It is unfortunately not possible to directly express the relationships between collateral characteristics and pricing through securitization, but we can illustrate some patterns using numerical calculations.

We can substitute the collateral par coupon expression in the ex-ante issuer's expected value. In 
the case of a standard structure with par-valued bonds, the gain to the issuer is

$$
V\left(g_{S S(0)}(X, V)\right)=\frac{y m}{1+y}
$$

and does not depend on the coupon. This expression only depends on the distribution of $V$ through $m$ and $D^{*}$ (on which $m$ depends).

In the case of an excess-spread structure we find that

$$
V\left(g_{X S(0)}(X, V)\right)=\frac{y m}{y p q+\mathbb{E}[V]} .
$$

The ratio, capturing how much more is gained by using excess-spread securitization over a standard structure, simplifies to

$$
\frac{V\left(g_{X S(0)}(X, V)\right)}{V\left(g_{S S(0)}(X, V)\right)}=\frac{1+y}{y p q+\mathbb{E}[V]} .
$$

As $y$ goes to zero, this quantity converges to $\frac{1}{\mathbb{E}[V]}$. Hence, if the discount rate of the issuer is close to that of the investors, being able to use an excess-spread structure is all the more so attractive as the collateral's expected recoveries are low. This pattern could explain how the use of excess-spread in the mortgage-backed securities allowed for an ever increasing preference for worse-quality underlying loans.

\section{$5.2 \quad$ Numerical Examples}

The interaction between the known credit risk $W$ and the privately observed credit risk $Z$ leads to complex calculations. In order to look at patterns in the pricing of collateral as driven by optimal structure execution, we need to make simplifying assumptions.

First, we assume that $V=Z W$, with $W$ independent from $Z$. For $W$ we choose a density such that $V$ has a uniform worst-case, and which gives more probability to high principal payment outcomes. It seems normal to expect that low loss observations are the most common. We pick the distribution

$$
\mu_{W}(w)=\frac{\beta+1}{1-w_{L}}\left(\frac{w-w_{L}}{1-w_{L}}\right)^{\beta} \mathbb{I}_{w \in\left[w_{L}, 1\right]}
$$

with $\beta \in(0,1)$ and $w_{L} \in[0,1]$, which we know satisfies the conditions of Proposition 3.2. The parameter $\beta$ is related to how more likely high loss observations are than low loss observations, 
and $w_{L}$ is the lowest possible amount of principal recovery. Then we have

$$
\mathbb{E}[W]=w_{L}+\left(1-w_{L}\right) \frac{\beta+1}{\beta+2}
$$

and for $d \in\left[w_{L}, 1\right]$,

$$
\mathbb{E}[W \wedge d]=d-\frac{d-w_{L}}{\beta+2}\left(\frac{d-w_{L}}{1-w_{L}}\right)^{\beta+1} .
$$

Hence

$$
\mathbb{E}\left[V \wedge D \mid Z=z_{L}\right]=z_{L} \mathbb{E}\left[W \wedge \frac{D}{z_{L}}\right]=D-\frac{D-z_{L} w_{L}}{\beta+2}\left(\frac{\frac{D}{z_{L}}-w_{L}}{1-w_{L}}\right)^{\beta+1}
$$

If we use $\mu_{Z}$ to write the density of $Z$ on $\left[z_{L}, 1\right]$, then the expression for $m$ cannot be readily simplified:

$$
m(D)=\left(\mathbb{E}\left[V \wedge D \mid Z=z_{L}\right]\right)^{\frac{1+y}{y}} \int_{z_{L}}^{1} d z \mu_{Z}(z)(\mathbb{E}[V \wedge D \mid Z=z])^{-\frac{1}{y}}
$$

Even with the simplest distribution assumption for $Z$, the first order condition for the maximum of $m$ cannot be solved formally for $D$, so we compute numerical solutions.

We pick the following parameters for the distribution of $W: \beta=0.5$ and $w_{L}=0.85$. For $Z$, we use a uniform distribution on $\left[z_{L}, 1\right]$ with $z_{L}=0.8$, and define $X=1+c T$, where $T$ is a uniform distribution on $\left[T_{L}, T_{H}\right]$ with $T_{L}=1$ and $T_{H}=10$. The coupon is set as $c=0.05$ initially but will later be determined implicitly so that the collateral is priced at par through an excess-spread structure execution.

We first look a the shape of $m(D)$ as a function of $D$, varying $w_{L}$ in Figure 4 , with all the other variables set as described above. We can see that the highest the maximum losses (or equivalently the lowest the minimum recovered principal) the less marked the optimal face value is, and the lower $m$ is at the optimum relative to $D$. In Figure 5, we fix $w_{L}$ and vary $z_{L}$ instead. We can see that the overall shape of $m$ then remains approximately the same when we change $z_{L}$, with the optimum $m$ being close to, and slightly lower than, $D$.

[Figure 4 about here.]

[Figure 5 about here.]

Numerically solving for the optimum $D^{*}$, we can plot that optimum as a function of the minimum recovery $w_{L}$ and varying the issuer's minimum observation $z_{L}$, as shown in Figure 6 . With our parameters, the optimum $D^{*}$ is not very sensitive to the collateral's worst-case outcome 
independent from the issuer's private observation. The influence of the worst-case as observed by the issuer is much stronger as we can see.

[Figure 6 about here.]

The resulting value to the issuer is shown in Figure 7. Here again, altering the distribution of $W$ does not have a very strong impact on the issuer's gain, although altering the worst-case outcome $z_{L}$ affects the issuer gain much more strongly.

[Figure 7 about here.]

With the optimal excess-spread structure, the quantity of senior securities sold $Q^{*}$ exhibits a more complex relationship to both $w_{L}$ and $z_{L}$, as shown in Figure 8.

[Figure 8 about here.]

As we have seen in Figure 7, with high-enough $w_{L}$ and $z_{L}$, the securitization gain to the issuer is in the order to 0.10 . Figure 9 shows that the limiting gain from a NIM resecuritization is fairly substantial in relation. We can also see that depending on $z_{L}$, there is a maximum to the value of a NIM as a function of the worst case $w_{L}$.

[Figure 9 about here.]

As the minimal recovery declines, and expected losses increase, the value to the issuer of an excess-spread securitization declines, but Figure 10 shows us that the slope of this decline is quite small. In effect, the collateral's coupon increases, for it to be par priced, as expected losses increase, and the excess-spread structure is able to make use of that higher coupon. A standard senior/subordination structure is not able to use the extra coupon, and its value to the issuer declines to a greater extent as expected losses increase.

[Figure 10 about here.]

Looking at the ratio of the value to the issuer of an excess-spread structure to the value of a standard structure as shown in Figure 10, we can see how par-priced collateral with a low credit quality will be much more efficiently securitized through an excess-spread structure.

[Figure 11 about here.] 


\section{Conclusion}

Securitization has been a subject of interest in the security design literature, and various models have been developed in order to explain why such transactions should produce senior securities and junior securities. However securitization structures are far more complex than a simple tranching by seniority.

Using a model inspired from the existing literature, specifically DeMarzo and Duffie (1999) and DeMarzo (2005), we have extended the analysis by considering that interest and principal should be separately contractible, and that the senior bonds in a securitization should be par-priced. This allowed us to derive optimal designs closely resembling actual securitization structures. Further, we showed that the resecuritization of residuals, in the form of NIMs or reremics, was optimal through a pooling effect comparable to that observed in DeMarzo (2005). Finally, we carried out a simple numerical analysis when the collateral is priced at par reflecting securitization execution. This has allowed us to illustrate how important resecuritization is, and also how more attractive an XS/OC structure was relative to a standard SI/SS as expected collateral losses increased. According to our analysis, the apparent complexity in excess-spread structure and in resecuritizations can be explained by a valid optimal design argument.

One important shortcoming in our analysis is that we have not addressed any of the complex dynamic aspects in securitization structures, as we only considered a two period economy. Extending security design models to a continuous time framework may make it possible to account for the optimality of some even more complex mechanisms, such as triggers and the timing of principal release.

\section{Appendix}

\subsection{Proof of Optimality of Dynamic Debt}

Proof. First, since we have assumed $Y$ has a uniform worst-case $z_{L}$, then using Proposition 10 from DeMarzo and Duffie (1999), we know that among all contracts of the form $g(Y)$, where interest is not contractible separately, the optimal contract has the form $g(Y)=Y \wedge D$ for some $D$.

Let us now show that optimal contracts have the form $f(x) k(v)=\left(x \wedge D_{0}\right)\left(v \wedge D_{1}\right)$. We consider a generic design with contractible interest $g(x, v) \leq x v$. We assume $g$ is increasing and continuous 
relative to $x$ and $v$. Given $g$, we define a dynamic debt contract $d(x, v)=\left(x \wedge D_{0}\right)\left(v \wedge D_{1}\right)$. Note that as a function of $D_{1}$ and as a function of $D_{0}, d$ is increasing and continuous. We set $D_{0}$ and $D_{1}$ such that

$$
\mathbb{E}\left[d(X, V) \mid Z=z_{L}\right]=\mathbb{E}\left[g(X, V) \mid Z=z_{L}\right] .
$$

Finding such values for $D_{0}$ and $D_{1}$ is possible since $d$ is continuous and increasing relative to them, and varying $D_{0}$ and $D_{1}, 0 \leq d(x, v) \leq x v$, and $0 \leq g(x, v) \leq x v$.

Then, comparing $V(d(X, V))$ and $V(g(X, V))$ only depends on the difference between $\mathbb{E}[d(X, V) \mid Z]$ and $\mathbb{E}[g(X, V) \mid Z]$. We write $e(x, v)=g(x, v)-d(x, v)$, which may be positive or negative, but is continuous in $x$ and in $v$. In addition, for $V \geq D_{1}$ and $X \geq D_{0}, e(x, v)$ is increasing in $v$. Hence there exists a continuous function $v^{*}$ depending on $g, D_{0}$ and $D_{1}$ such that $v>v^{*}(x)$ is equivalent to $e(x, v)>0$.

Now, we write

$$
\begin{aligned}
\mathbb{E}[e(X, V) \mid Z=z] & =\mathbb{E}\left[\mathbb{I}_{e(X, V)>0} e(X, V) \mid Z=z\right]+\mathbb{E}\left[\mathbb{I}_{e(X, V) \leq 0} e(X, V) \mid Z=z\right] \\
& =\mathbb{E}\left[\mathbb{I}_{V>v^{*}(X)} e(X, V) \nu_{z}(V) \mid Z=z_{L}\right] \\
& +\mathbb{E}\left[\mathbb{I}_{V \leq v^{*}(X)} e(X, V) \nu_{z}(V) \mid Z=z_{L}\right]
\end{aligned}
$$

where we know that $\nu_{z}$ is increasing. Noting that the second term in the sum is negative, we can write:

$$
\mathbb{E}[e(X, V) \mid Z=z] \geq \mathbb{E}\left[\nu_{z}\left(v^{*}(X)\right)\right] \mathbb{E}\left[e(X, V) \mid Z=z_{L}\right]
$$

However, we set $D_{0}$ and $D_{1}$ so that $\mathbb{E}\left[e(X, V) \mid Z=z_{L}\right]=0$ and therefore $\mathbb{E}[e(X, V) \mid Z=z] \geq 0$. As a consequence, given a generic contract $g$, we have determined a superior contract of the form $\left(x \wedge D_{0}\right)\left(V \wedge D_{1}\right)$.

Now, we consider contracts of this form $g(x, v)=f(x) k(v)$, with $f(x)=\left(x \wedge D_{0}\right)$ and $k(v)=$ $\left(v \wedge D_{1}\right)$. We can write

$$
V(g(X, V))=\frac{y}{1+y} \mathbb{E}[f(X)] \mathbb{E}\left[k(V) \mid Z=z_{L}\right] \mathbb{E}\left[\left(\frac{\mathbb{E}\left[k(V) \mid Z=z_{L}\right]}{\mathbb{E}[k(V) \mid Z]}\right)^{\frac{1}{y}}\right]
$$

This value is maximal for $f$ set as the identity, and therefore the optimal contract takes the form: $X(V \wedge D)$ for some $D$ that maximizes $V(X(V \wedge D))$. 


\subsection{Conditions for Uniform Worst-Case}

Proof. We want to show that $A Z$ has a uniform worst-case $z_{L}$ for any independent random variable $A$ with distribution $\mu_{A}$ verifying the conditions in the Proposition.

In this case we can write the conditional expectation $\mathbb{E}[g(A Z) \mid Z=z]$ as follows

$$
\begin{aligned}
\int g(y) \mu(y, z) d y & =\int g(z a) \mu_{A}(a) d a \\
& =\int g(y) \mu_{A}\left(\frac{y}{z}\right) \frac{d y}{z} \\
& =\int g(y) \frac{z_{L} \mu_{A}\left(\frac{y}{z}\right)}{z \mu_{A}\left(\frac{y}{z_{L}}\right)} \mu_{A}\left(\frac{y}{z_{L}}\right) \frac{d y}{z_{L}} \\
& =\int g\left(z_{L} a\right) \frac{z_{L} \mu_{A}\left(\frac{z_{L}}{z} a\right)}{z \mu_{A}(a)} \mu_{A}(a) d a .
\end{aligned}
$$

And therefore the Radon-Nikodym derivative is

$$
\nu_{z}(y)=\frac{z_{L} \mu_{A}\left(\frac{y}{z}\right)}{z \mu_{A}\left(\frac{y}{z_{L}}\right)} .
$$

Since the $z_{L}$ is a lower bound of $Z$, any value $z$ in the domain of $Z$ verifies $z \geq z_{L}$ and $\frac{z}{z_{L}}>1$. For $\nu_{z}(y)$ to be increasing, the first derivative needs to be positive. If we write $a=\frac{y}{z}$ and $\alpha a=\frac{y}{z_{L}}$ for simplicity (with $\alpha>1$ ), the condition is:

$$
\mu_{A}^{\prime}(a) \mu_{A}(\alpha a) \geq \alpha \mu_{A}(a) \mu_{A}^{\prime}(\alpha a) .
$$

Now we show that if $\mu_{A}$ is log-concave, along with another condition, then the condition

$$
\frac{\mu_{A}^{\prime}(a)}{\mu_{A}(a)} \geq \alpha \frac{\mu_{A}^{\prime}(\alpha a)}{\mu_{A}(\alpha a)}
$$

is verified.

Assume that $\mu_{A}$ is log-concave. In this case $\frac{\mu_{A}^{\prime}}{\mu_{A}}$ is decreasing. We also assume the ratios are defined and vanish at zero, so that $\frac{\mu_{A}^{\prime}(a)}{\mu_{A}(a)}$ goes to zero when $a$ goes to zero. With $a=0$, we see that this is necessary for the inequality to be verified, otherwise we would arrive to $\alpha \leq 1$. Therefore we see that $\frac{\mu_{A}^{\prime}}{\mu_{A}} \leq 0$, so this ratio must be decreasing and also negative. 
Now, we note that thanks to log-concavity

$$
\frac{\mu_{A}^{\prime}(a)}{\mu_{A}(a)} \geq \frac{\mu_{A}^{\prime}(\alpha a)}{\mu_{A}(\alpha a)}
$$

but both sides of this inequality are negative so

$$
\frac{\mu_{A}^{\prime}(\alpha a)}{\mu_{A}(\alpha a)} \geq \alpha \frac{\mu_{A}^{\prime}(\alpha a)}{\mu_{A}(\alpha a)}
$$

and the condition is verified.

If on the other hand we do not assume that $\frac{\mu_{A}^{\prime}(a)}{\mu_{A}(a)}$ goes to zero when $a$ goes to zero, then we need to assume that $\frac{\mu_{A}^{\prime}(a)}{\mu_{A}(a)}$ goes to infinity when $a$ goes to zero, again so that $\alpha \leq 1$ is not implied. In this case, $\frac{\mu_{A}^{\prime}}{\mu_{A}} \geq 0$ at least on some of its domain. In the cases where this quantity is negative, we can infer the condition as we have above. Where $\frac{\mu_{A}^{\prime}}{\mu_{A}}$ is positive, and $\mu_{A}^{\prime}$ is positive, the condition of log-concavity is not sufficient and we need a stronger specific assumption, that:

$$
\frac{\mu_{A}^{\prime}(a)}{\mu_{A}(a)} \geq \alpha \frac{\mu_{A}^{\prime}(\alpha a)}{\mu_{A}(\alpha a)}
$$

This implies that the second derivative of $\frac{\mu_{A}^{\prime}(a)}{\mu_{A}(a)}$ be negative enough.

Hence, the variables of the form $Z A$ admit a uniform worst-case equal to inf $Z$ when $A$ has a log-concave distribution $\mu_{A}$ either with $\frac{\mu_{A}^{\prime}(a)}{\mu_{A}(a)}$ vanishing in zero, or if $\frac{\mu_{A}^{\prime}(a)}{\mu_{A}(a)}$ goes to infinity in zero, then the stronger condition that $\frac{\mu_{A}^{\prime}(a)}{\mu_{A}(a)} \geq \alpha \frac{\mu_{A}^{\prime}(\alpha a)}{\mu_{A}(\alpha a)}$ is required.

\subsection{Optimality of NIM Securitization}

Proof. We follow the outline of the proof of Theorem II in DeMarzo (2005).

We know that $V=W Z$ and $Y=X W Z$ have $z_{L}$ as a uniform worst case, and so does $\left(Z X W-D^{*}\right) \mathbb{I}_{Z X W-D^{*} \geq 0}$ as an increasing function. Thanks to the log-concavity assumption, $\sum_{i}\left(Z_{i} X_{i} W_{i}-D^{*}\right) \mathbb{I}_{Z_{i} X_{i} W_{i}-D^{*} \geq 0}$ also admits a uniform worst-case relative to the $Z_{i}$ and since the distribution is the same for all $i$ it is equal to $z_{L}$.

Hence, thanks to Proposition ?? we know that among all security designs of the form

$$
g\left(\frac{1}{n} \sum_{i \geq 1}^{n}\left(Z_{i} X_{i} W_{i}-D^{*}\right) \mathbb{I}_{Z_{i} X_{i} W_{i}-D^{*} \geq 0}\right),
$$


a standard debt contract

$$
\begin{aligned}
& g_{N I M}^{d}\left(\frac{1}{n} \sum_{i \geq 1}^{n}\left(Z_{i} X_{i} W_{i}-D^{*}\right) \mathbb{I}_{Z_{i} X_{i} W_{i}-D^{*} \geq 0}\right) \\
& =\left(\frac{1}{n} \sum_{i \geq 1}^{n}\left(Z_{i} X_{i} W_{i}-D^{*}\right) \mathbb{I}_{Z_{i} X_{i} W_{i}-D^{*} \geq 0}\right) \wedge d
\end{aligned}
$$

is optimal.

Let us now define

$$
\begin{aligned}
j_{n}(d, z) & =\mathbb{E}\left[\left(\frac{1}{n} \sum_{i \geq 1}^{n}\left(Z_{i} X_{i} W_{i}-D^{*}\right) \mathbb{I}_{Z_{i} X_{i} W_{i}-D^{*} \geq 0}\right) \wedge d \mid \forall i \leq n: Z_{i}=z\right] \\
& =\mathbb{E}\left[\left(\frac{1}{n} \sum_{i \geq 1}^{n}\left(z X_{i} W_{i}-D^{*}\right) \mathbb{I}_{z X_{i} W_{i}-D^{*} \geq 0}\right) \wedge d\right] .
\end{aligned}
$$

As $z_{L}$ is the uniform worst case for all the $Z_{i} X_{i} W_{i}$ we have

$$
\begin{aligned}
& \inf _{\left(z_{i}\right)_{1 \leq i \leq n}} \mathbb{E}\left[\left(\frac{1}{n} \sum_{i \geq 1}^{n}\left(Z_{i} X_{i} W_{i}-D^{*}\right) \mathbb{I}_{Z_{i} X_{i} W_{i}-D^{*} \geq 0}\right) \wedge d \mid \forall i \leq n: Z_{i}=z_{i}\right] \\
& =\mathbb{E}\left[\left(\frac{1}{n} \sum_{i \geq 1}^{n}\left(Z_{i} X_{i} W_{i}-D^{*}\right) \mathbb{I}_{Z_{i} X_{i} W_{i}-D^{*} \geq 0}\right) \wedge d \mid \forall i \leq n: Z_{i}=z_{L}\right] \\
& =j_{n}\left(d, z_{L}\right) .
\end{aligned}
$$

The value to the issuer of the contract $g_{N I M}^{d}$ is therefore as follows (we did not write the full random variable expression on the left-hand side for brevity):

$$
\begin{aligned}
& V\left(g_{N I M}^{d}\right)=\frac{y}{1+y} j_{n}\left(d, z_{L}\right) \\
& \mathbb{E}\left[\left(\frac{j_{n}\left(d, z_{L}\right)}{\mathbb{E}\left[\left(\frac{1}{n} \sum_{i \geq 1}^{n}\left(Z_{i} X_{i} W_{i}-D^{*}\right) \mathbb{I}_{Z_{i} X_{i} W_{i}-D^{*} \geq 0}\right) \wedge d \mid\left(Z_{i}\right)_{1 \leq i \leq n}\right]}\right)^{\frac{1}{y}}\right] .
\end{aligned}
$$

The $X_{i} W_{i}$ are independent and identically distributed, so thanks to the weak law of large numbers, we know that $j_{n}(d, z)$ converges and

$$
\lim _{n \rightarrow \infty} j_{n}(d, z)=\mathbb{E}\left[\left(z W X-D^{*}\right) \mathbb{I}_{z W X-D^{*} \geq 0}\right] \wedge d
$$


We define $r_{L}=\mathbb{E}\left[\left(z_{L} W X-D^{*}\right) \mathbb{I}_{z_{L} W X-D^{*} \geq 0}\right]$, so that if we set $d=r_{L}$ then

$$
\lim _{n \rightarrow \infty} j_{n}\left(r_{L}, z\right)=\mathbb{E}\left[\left(z W X-D^{*}\right) \mathbb{I}_{z W X-D^{*} \geq 0}\right] \wedge r_{L}=r_{L},
$$

since $z_{L}$ is the uniform worst case of $W X Z$.

In addition, note that

$$
r_{L} \geq \mathbb{E}\left[\left(\frac{1}{n} \sum_{i \geq 1}^{n}\left(Z_{i} X_{i} W_{i}-D^{*}\right) \mathbb{I}_{Z_{i} X_{i} W_{i}-D^{*} \geq 0}\right) \wedge r_{L} \mid\left(Z_{i}\right)_{1 \leq i \leq n}\right]
$$

as well as

$$
\mathbb{E}\left[\left(\frac{1}{n} \sum_{i \geq 1}^{n}\left(Z_{i} X_{i} W_{i}-D^{*}\right) \mathbb{I}_{Z_{i} X_{i} W_{i}-D^{*} \geq 0}\right) \wedge r_{L} \mid\left(Z_{i}\right)_{1 \leq i \leq n}\right] \geq j_{n}\left(r_{L}, z_{L}\right)
$$

Since $j_{n}\left(r_{L}, z_{L}\right)$ goes to $r_{L}$, then the conditional expectation converges to $r_{L}$ as well.

As a result, we see that

$$
\lim _{n \rightarrow \infty} V\left(g_{N I M}^{r_{L}}\right)=\frac{y}{1+y} r_{L}
$$

Now let us verify that setting $d$ to $r_{L}$ is the optimal contract. If $d>r_{L}$ then we know that the value to the issuer $V\left(g_{N I M}^{d}\right)$ verifies

$$
V\left(g_{N I M}^{d}\right) \leq \frac{y}{1+y} j_{n}\left(d, z_{L}\right)
$$

and $j_{n}\left(d, z_{L}\right)$ goes to $d \wedge r_{L}$, which by our assumption is $r_{L}$. If $d<r_{L}$, then we can also write $V\left(g_{N I M}^{d}\right) \leq \frac{y}{1+y} j_{n}\left(d, z_{L}\right)$ but now $j_{n}\left(d, z_{L}\right)$ goes to $d$ which is smaller than $r_{L}$. Hence setting the NIM size to $r_{L}$ is optimal at the limit.

\section{References}

Begley, Taylor A., and Amiyatosh Purnanandam. 2017. "Design of Financial Securities: Empirical Evidence from Private-Label RMBS Deals." The Review of Financial Studies 30 (1): $120-61$.

Boot, Arnoud, and Anjan Thakor. 1993. "Security Design." Journal of Finance 48 (4): 1349-78. Bougheas, Spiros. 2014. "Pooling, Tranching, and Credit Expansion." Oxford Economic Papers 
66 (2): $557-79$.

Crawford, Alec. 2007. "A Guide to U.s. MBS.” Research Report. RBS Greenwich Capital.

Dang, Tri Vi, Gary Gorton, and Bengt Holmström. 2015. "The Information Sensitivity of a Security." Working Paper. Columbia University.

DeMarzo, Peter. 2005. "The Pooling and Tranching of Securities: A Model of Informed Intermediation." The Review of Financial Studies 18 (1): 1-35.

DeMarzo, Peter, and Darrell Duffie. 1999. "A Liquidity-Based Model of Security Design." Econometrica 67 (1): 65-100.

Diamond, Douglas. 1993. "Seniority and Maturity of Debt Contracts." Journal of Financial Economics 33 (3): 341-68.

Fabozzi, Frank J., ed. 2016. The Handbook of Mortgage-Backed Securities, 7th Edition. Oxford University Press.

Fender, Ingo, and Janet Mitchell. 2009. "Incentives and Tranche Retention in Securitisation: A Screening Model." CEPR Discussion Papers 7483. C.E.P.R. Discussion Papers.

Franke, Günter, Markus Herrmann, and Thomas Weber. 2012. "Loss Allocation in Securitization Transactions." Journal of Financial and Quantitative Analysis 47 (5): 1125-53.

Gaur, Vishal, Sridhar Seshadri, and Marti G. Subrahmanyam. 2003. "Market Incompleteness and Super Value Additivity: Implications for Securitization." LSE Research Online Documents on Economics OM-2005-07. New York University.

Gauthier, Laurent. 2002. "The Secrets of NIMs." Research Report. UBS. 2004. "An Introduction to CMOs." Research Report. Morgan Stanley.

Gauthier, Laurent, and Tom Zimmerman. 2002a. "Introduction to the Home-Equity Loan Market." Research Report. UBS. 2002b. "The Analysis of Non-Agency Mortgage Credit." Research Report. UBS.

Gorton, Gary. 2008. "The Subprime Panic.” NBER Working Papers 14398. National Bureau of Economic Research, Inc.

Gorton, Gary, and Nicholas Souleles. 2007. "Special Purpose Vehicles and Securitization." In 
The Risks of Financial Institutions, 549-602. National Bureau of Economic Research, Inc.

Hayre, Lakhbir, and Robert Young. 2004. "Guide to Mortgage-Backed Securities." Research Report. Citigroup.

Hein, Julia. 2009. "Four Essays on Credit Securitization, Phd Thesis." University of Konstanz. Leland, Hayne, and David H. Pyle. 1977. "Informational Asymmetries, Financial Structure, and Financial Intermediation." Journal of Finance 32 (2): 371-87.

McConnell, John, and Stephen Buser. 2011. "The Origins and Evolution of the Market for Mortgage-Backed Securities." Annual Review of Financial Economics, no. 3: 173-92.

Myers, Stewart C., and Nicholas S. Majluf. 1984. "Corporate Financing and Investment Decisions When Firms Have Information That Investors Do Not Have." Journal of Financial Economics 13 (2): 187-221.

Park, Sunyoung. 2013. "The Design of Subprime Mortgage-Backed Securities and Information Insensitivity." International Economic Journal 27 (2): 249-84.

Plantin, Guillaume. 2003. "Tranching.” LSE Research Online Documents on Economics. London School of Economics; Political Science, LSE Library. 


\section{List of Figures}

1 A Comparison of Excess-Spread and Shifting-Interest/Senior-Sub Structures . . . 34

2 Example Reremic Structure . . . . . . . . . . . . . . . . . . . . 34

3 Example NIM Structure . . . . . . . . . . . . . . . . . . . . 35

$4 \quad m(D)$ vs. Face Value $D$ Varying $w_{L} \ldots \ldots \ldots \ldots \ldots \ldots \ldots$

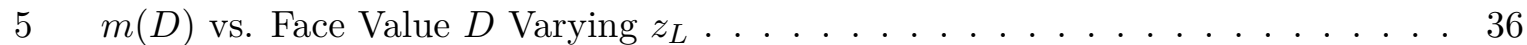

$6 \quad$ Optimal Face Value $D^{*}$ as a Function of $w_{L}$ Varying $z_{L} \ldots \ldots \ldots \ldots$

$7 \quad$ Maximal Isuer Value as a Function of $w_{L}$ Varying $z_{L} \ldots \ldots \ldots \ldots$. . . . . . 37

8 Optimal Quantity Sold as a Function of $w_{L}$ Varying $z_{L} \ldots \ldots \ldots$. . . . . 37

$9 \quad$ Gain From NIM Issuance as a Function of $w_{L}$ Varying $z_{L} \ldots \ldots \ldots$

10 Gain From XS Structure Off Par Price Collateral as a Function of $w_{L}$ Varying $z_{L} \quad 38$

11 Ratio of Gains from XS Structure to SS Structure Off Par Price Collateral as a

Function of $w_{L}$ Varying $z_{L} \ldots \ldots \ldots \ldots \ldots$ 


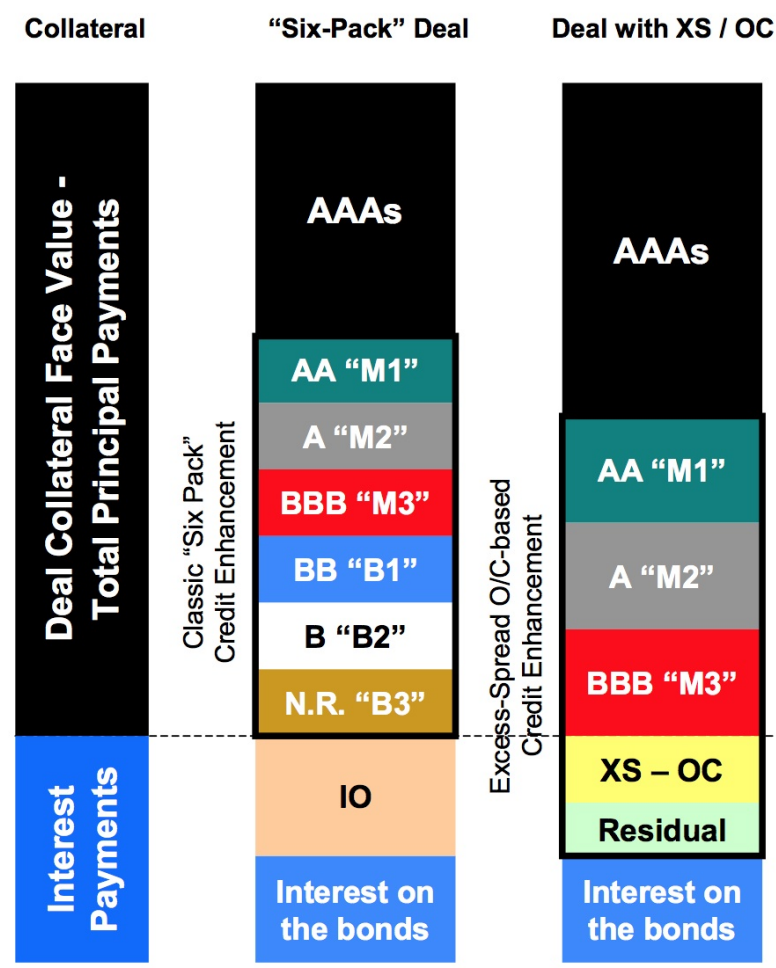

Figure 1: A Comparison of Excess-Spread and Shifting-Interest/Senior-Sub Structures

\begin{tabular}{|} 
Total face value $\mathbf{=} \mathbf{\$ 1 0 0 m m}$ \\
\hline Portfolio of Bonds \\
\hline NR Jumbos & NR Alt-As \\
\hline B Jumbos & B Alt-As \\
\hline BB Jumbos & BB Alt-As \\
\hline IG Jumbos & IG Alt-As \\
\hline Seasoned & Recent \\
\hline Etc... \\
\hline
\end{tabular}

\begin{tabular}{|c|c|}
\hline $\begin{array}{l}\text { All cash flows: } \\
\text { Principal + Interest - Losses }\end{array}$ & Turbo Sequential \\
\hline $\begin{array}{r}\text { NOTES face value } \\
=\$ 85 \mathrm{~mm}\end{array}$ & $\begin{array}{l}\text { BBB-rated NOTES } \\
\text { [1] All cash flows first pay } \\
\text { interest on the NOTES, then } \\
\text { principal on the NOTES }\end{array}$ \\
\hline $\begin{array}{r}\text { NOTES protection: } \\
\$ 15 \mathrm{~mm} \text { of O/C } \\
+ \text { Excess Spread }\end{array}$ & $\begin{array}{l}\text { Unrated RESIDUAL } \\
\text { [2] When all the outstanding } \\
\text { principal on the NOTES has } \\
\text { been paid, the cash flows go } \\
\text { to the RESIDUAL }\end{array}$ \\
\hline
\end{tabular}

Figure 2: Example Reremic Structure 


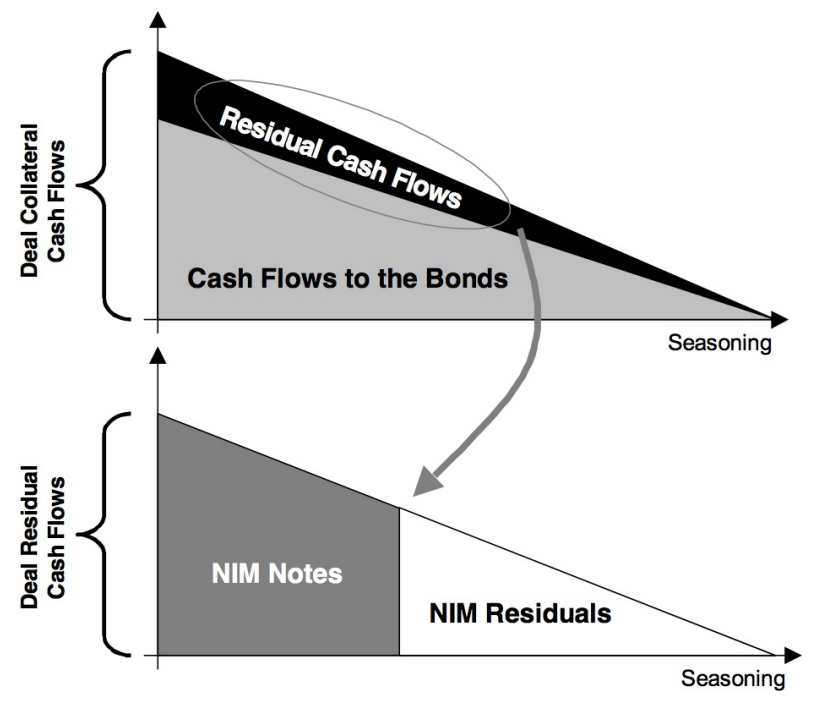

Figure 3: Example NIM Structure

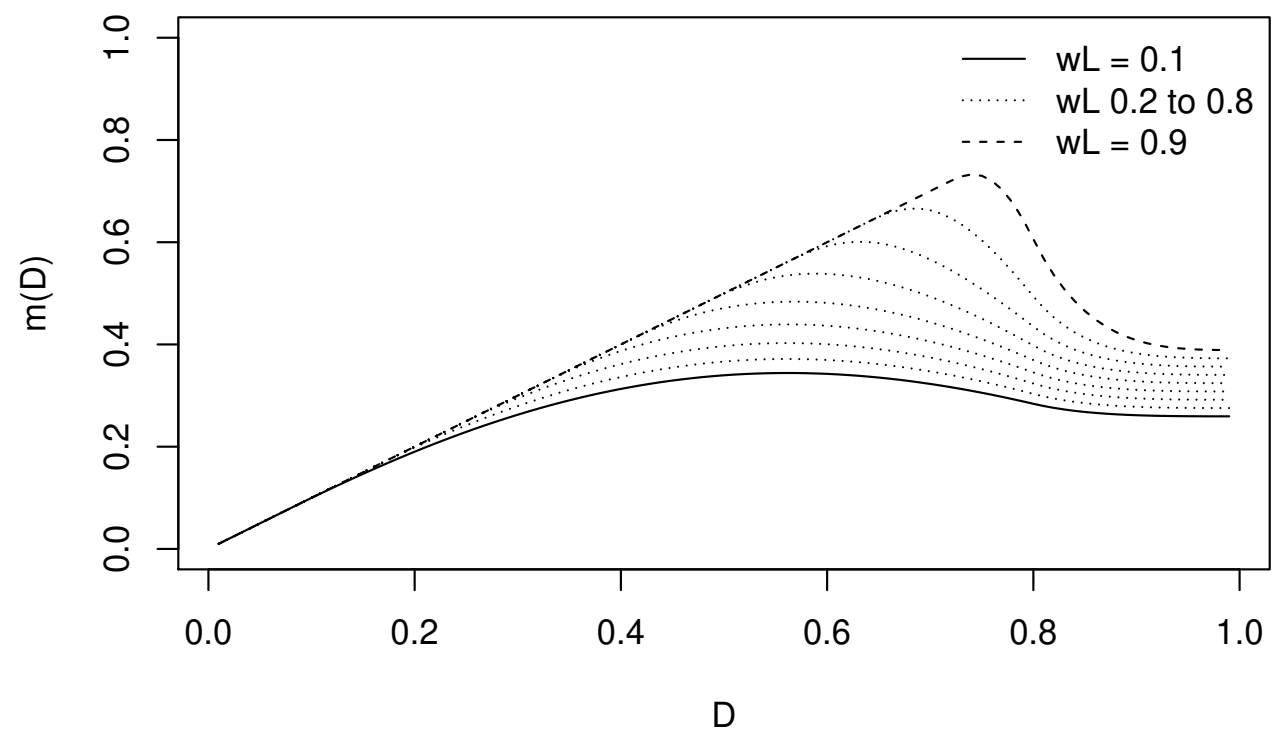

Figure 4: $m(D)$ vs. Face Value $D$ Varying $w_{L}$ 


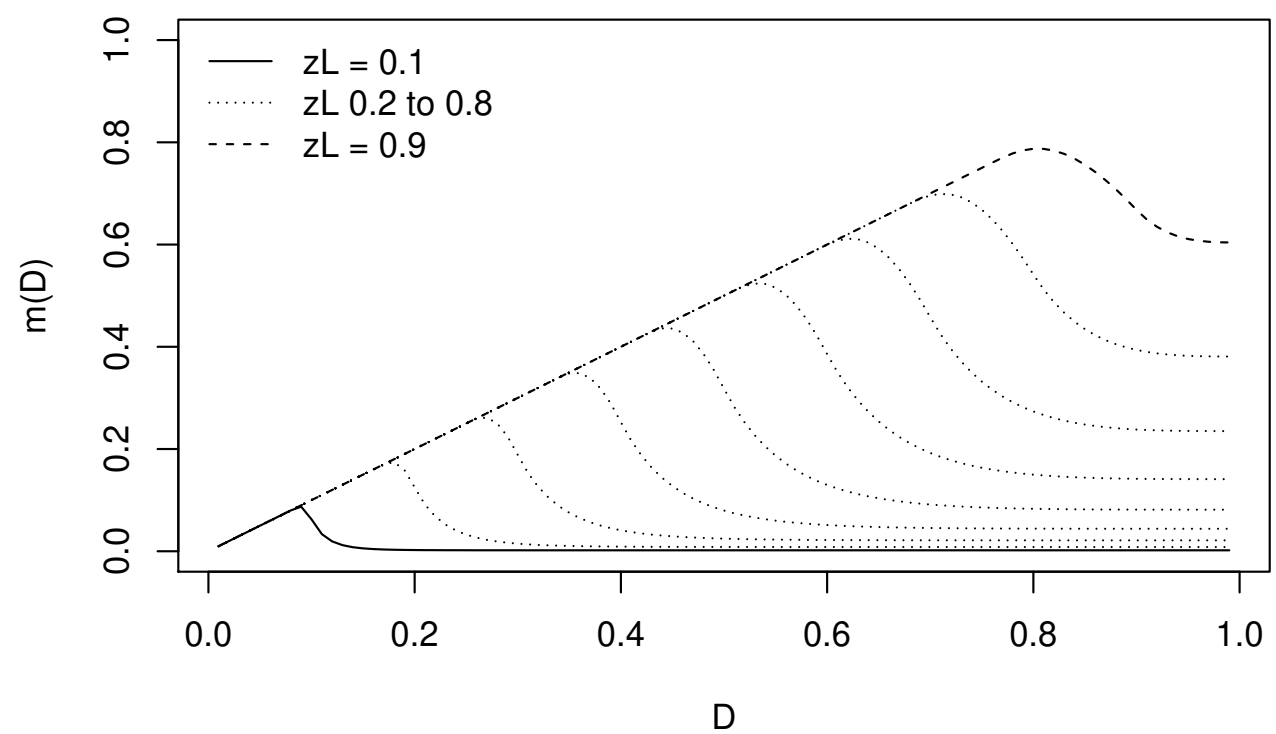

Figure 5: $m(D)$ vs. Face Value $D$ Varying $z_{L}$

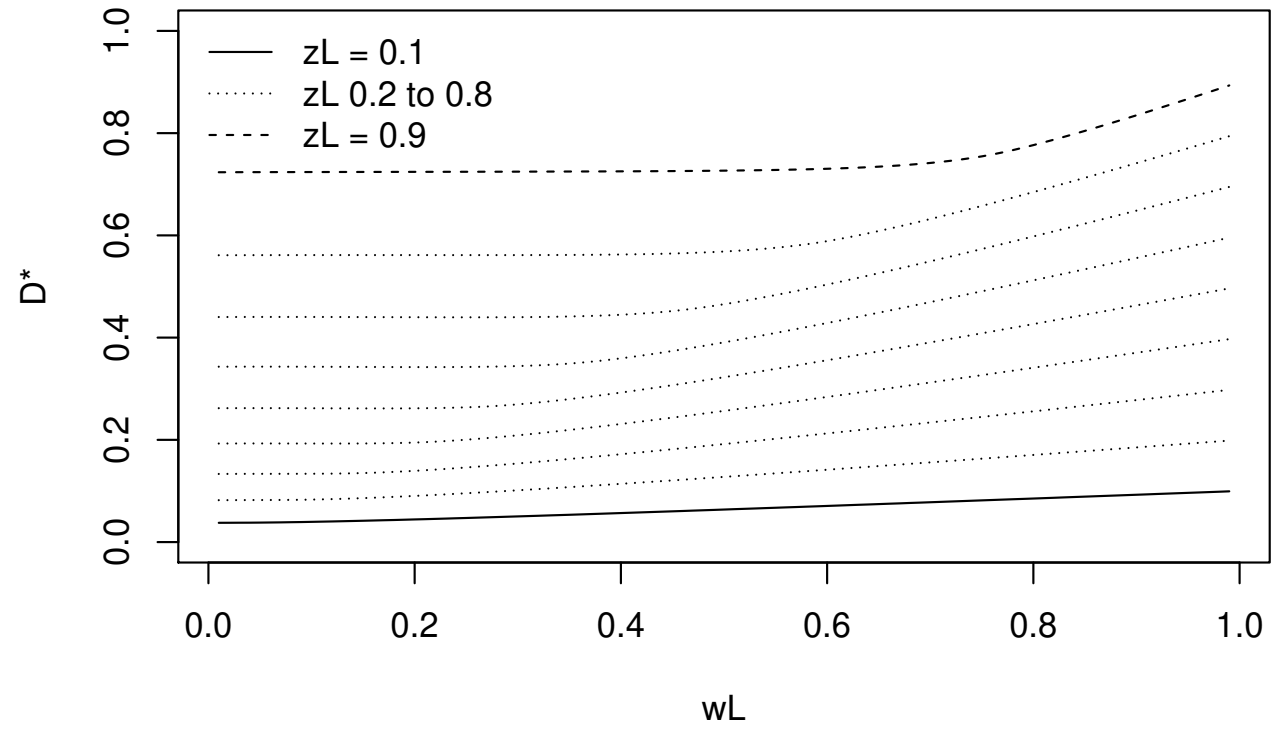

Figure 6: Optimal Face Value $D^{*}$ as a Function of $w_{L}$ Varying $z_{L}$ 


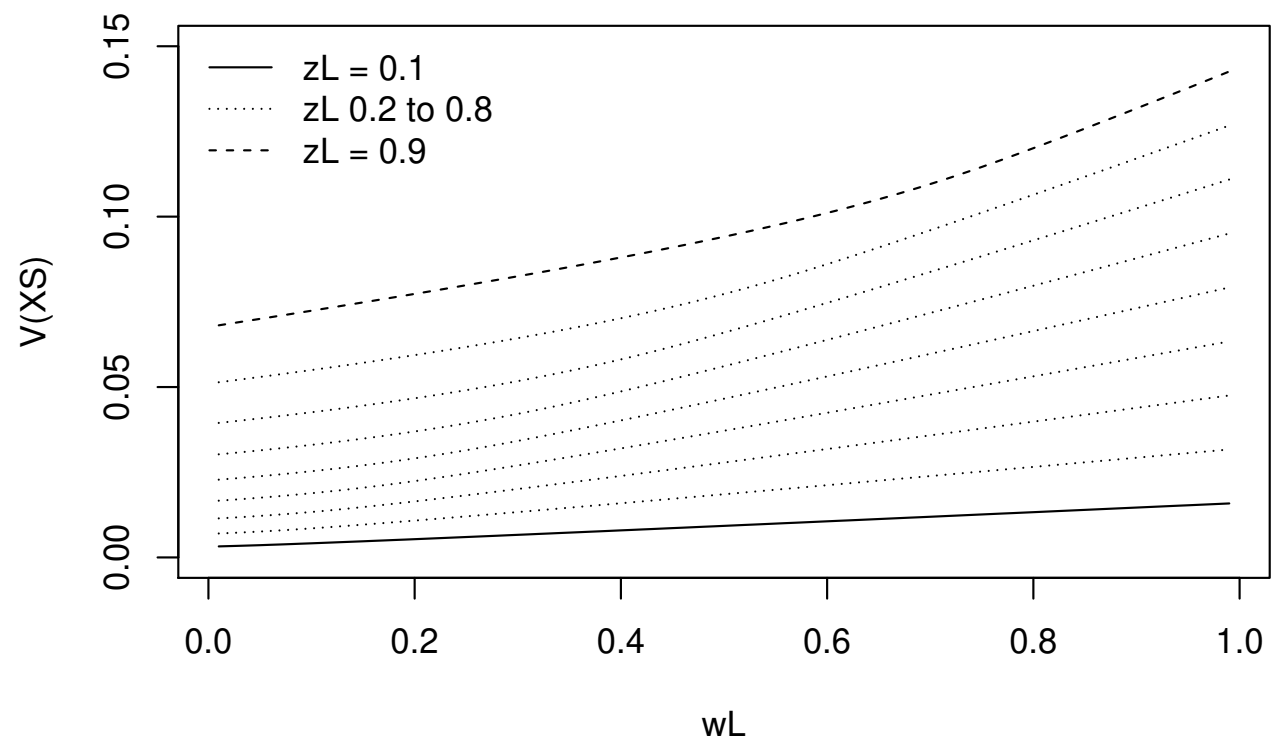

Figure 7: Maximal Isuer Value as a Function of $w_{L}$ Varying $z_{L}$

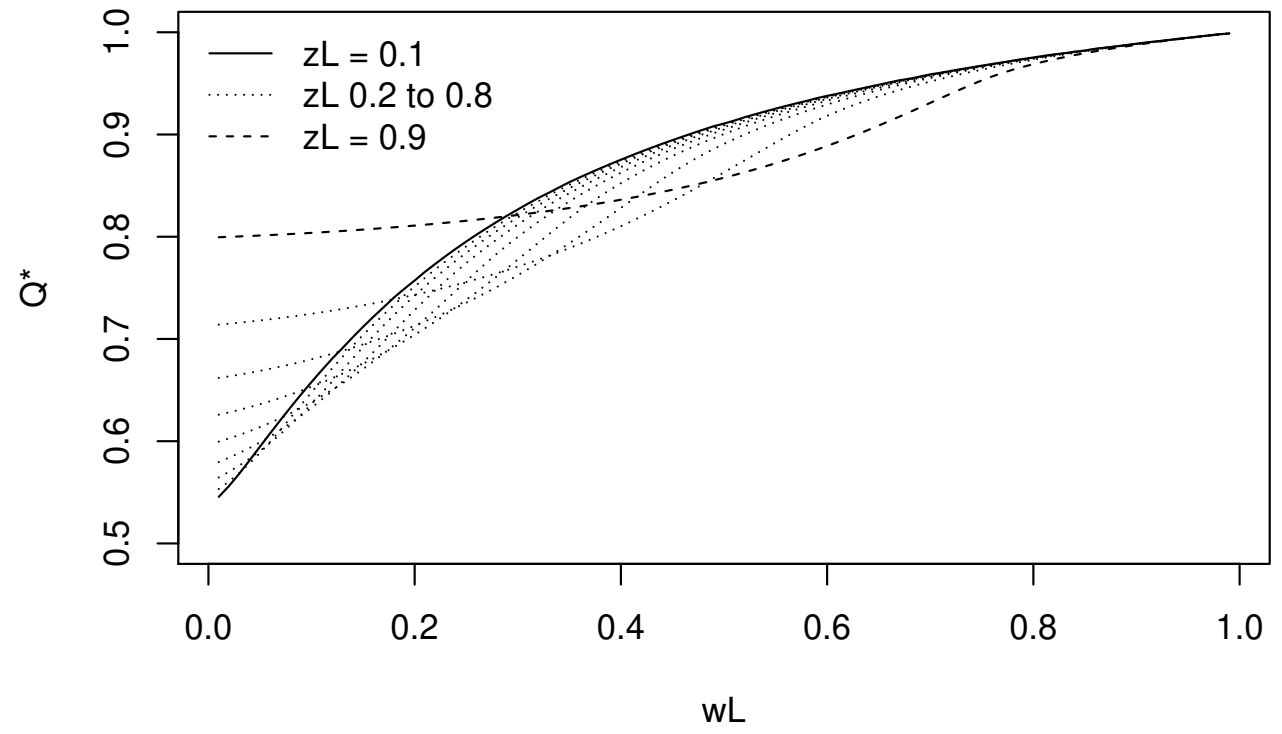

Figure 8: Optimal Quantity Sold as a Function of $w_{L}$ Varying $z_{L}$ 


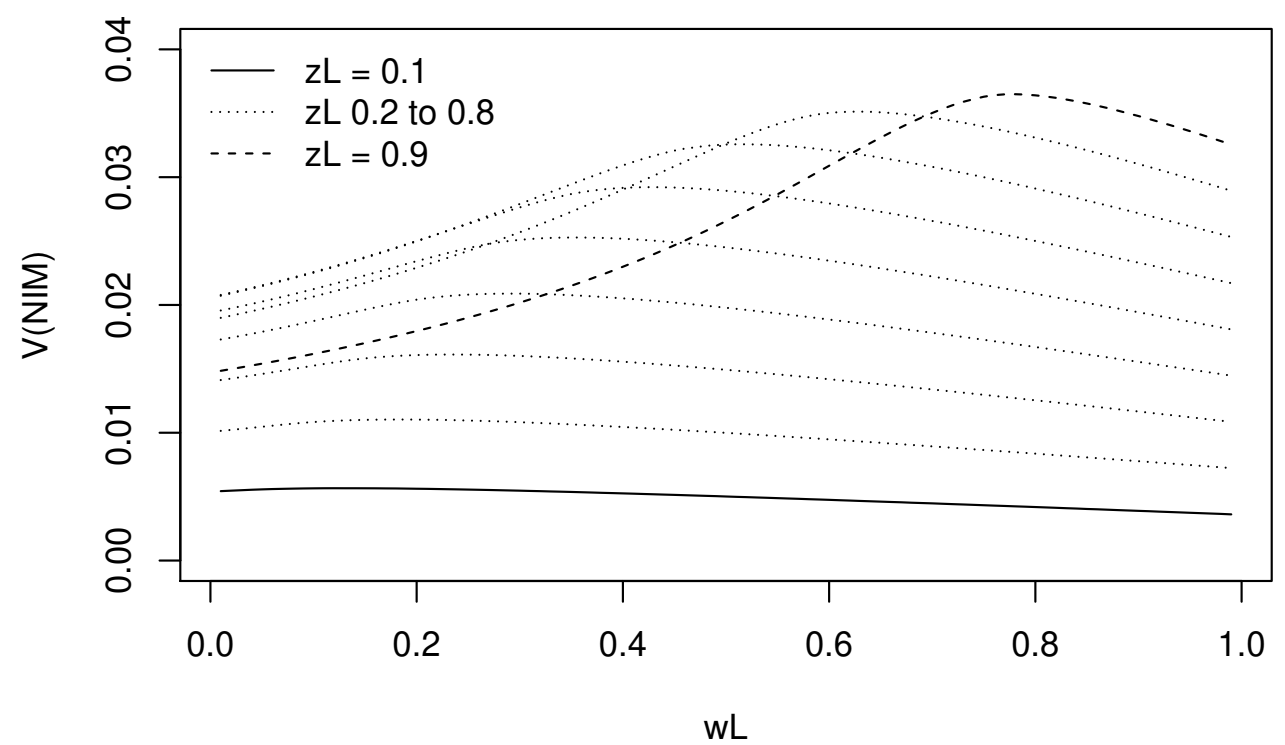

Figure 9: Gain From NIM Issuance as a Function of $w_{L}$ Varying $z_{L}$

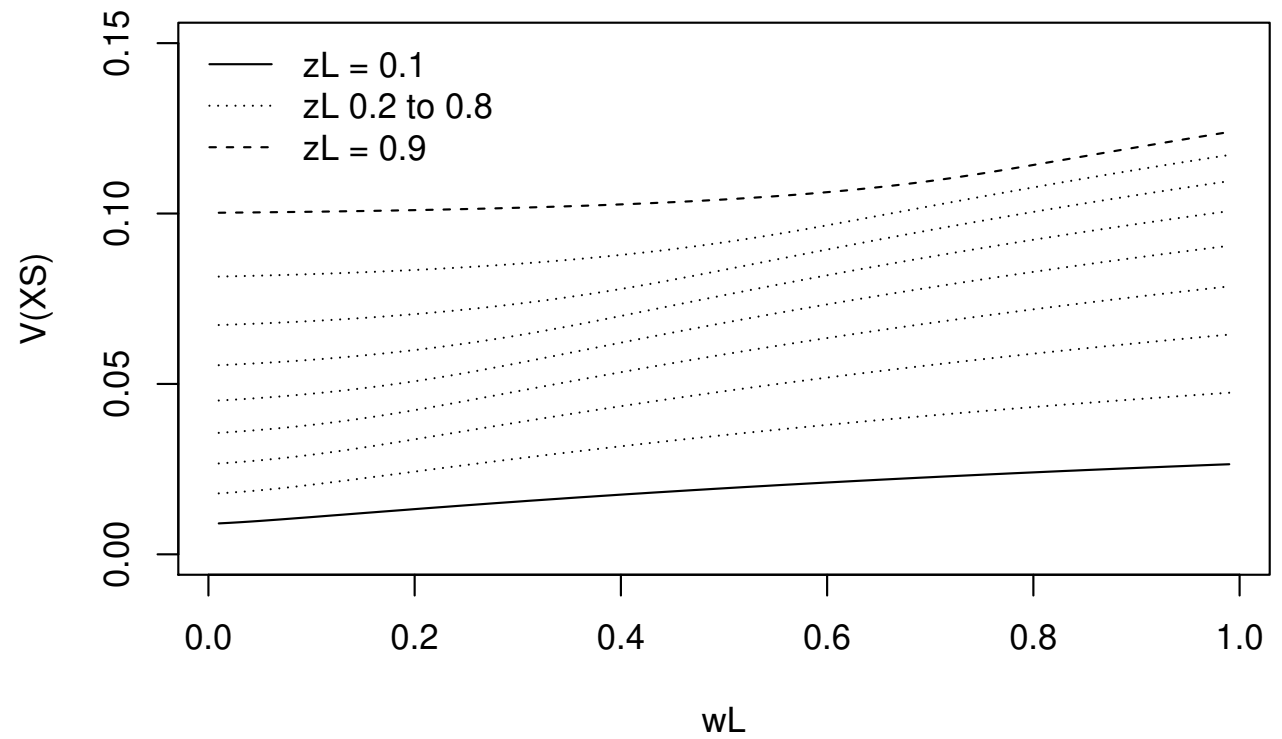

Figure 10: Gain From XS Structure Off Par Price Collateral as a Function of $w_{L}$ Varying $z_{L}$ 


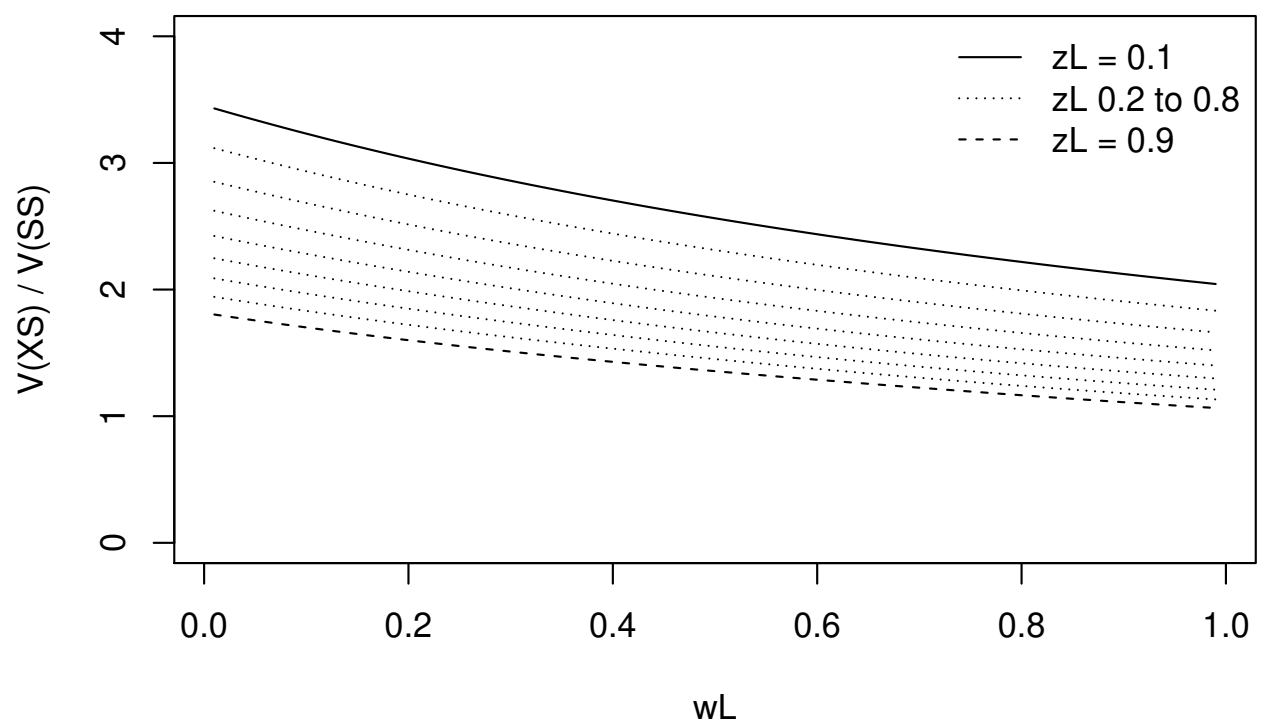

Figure 11: Ratio of Gains from XS Structure to SS Structure Off Par Price Collateral as a Function of $w_{L}$ Varying $z_{L}$ 